\title{
Dicing of Thin Silicon Wafers with Ultra-Short Pulsed Lasers in the Range from 200 fs up to $10 \mathrm{ps}$
}

\author{
C. Fornaroli ${ }^{1}$, J. Holtkamp ${ }^{1}$, Arnold Gillner ${ }^{1}$ \\ 1- Fraunhofer ILT, Steinbachstrasse 15, 52074 Aachen, Germany
}

Christian.Fornaroli@ilt.fraunhofer.de

\begin{abstract}
In this paper the influence of the pulse duration in a range of $200 \mathrm{fs}$ up to $10 \mathrm{ps}$ on the cutting process of thin silicon is investigated. The experiments are carried out with a Light Conversion Pharos with various pulse durations between $200 \mathrm{fs}$ and $10 \mathrm{ps}$. The laser is operated at the second harmonic wavelength of $515 \mathrm{~nm}$ and a repetition rate of $200 \mathrm{kHz}$. Although the ablation threshold, which is determined with the method of Liu [1], amounts $0,27 \mathrm{~J} / \mathrm{cm}^{2}$ for $0,2,1$ and $10 \mathrm{ps}$, one can find extremely different ablation behavior during the cutting experiments. It turns out that independent from pulse energy and pulse overlap, the shortest pulse duration at 200 fs leads to a deeper cutting depth and furthermore to a higher cutting speed. This difference can be explained with higher pulse peak intensities and shorter interaction time between laser and material for the $200 \mathrm{fs}$ pulse duration. Nevertheless the general ablation geometry is very similar for all tested pulse durations. We explain this with a simple geometric model. This model considers the projection from a Gaussian beam profile in an existing cutting kerf.
\end{abstract}

DOI: $10.2961 / \mathrm{jlmn} .2015 .02 .0022$

Keywords: Ultra-fast, silicon, grooving, dicing, femto second, pico seconds

\section{Introduction and motivation}

Currently, electrical semiconductor components such as LEDs, solar cells or transistors are commonly produced in a batch process. This way, many identical components can be processed in parallel on one big wafer; subsequently, each chip has to be singulated. In contrast to state-of-the-art technologies like blade sawing and nanosecond-based laser processes, laser dicing with ultra-short pulsed lasers offers fundamental advantages [2]. Due to the short interaction time between laser and material, small kerf widths, marginal heat affected zones and minimal edge damaging are attainable. While a reduction of the kerf width leads to a higher yield per wafer, minimal thermal and mechanical damage increases the breaking strength of each die [3].

Mechanical sawing with diamond blades has been used for a long time, but as the wafer material gets thinner and the chip size smaller, this classical process can be replaced by laser-based dicing processes. In particular, the mechanical load and the relatively large kerf width are serious disadvantages of a mechanical dicing process. A reduction of the kerf width leads to a much higher yield of chips per wafer and, therefore, to increasing efficiency and conserving resources at the same time. Furthermore, diamond blades are not suitable to cut thin wafers in the range of 100 $\mu \mathrm{m}$ or less, because they cannot resharpen themselves at the thin wafer edge.

Several laser dicing technologies are currently available on the market:

- $\quad$ Full cut with ns laser at UV-Wavelength [4]

- Stealth dicing with ns at IR-Wavelength [5]

- Full cut with a water-jet guided ns laser at IRWavelength [6]
Although these developed technologies overcome some of the problems connected with a mechanical sawing process, each one can be optimized. For example, the kerf loss in a ns-laser cutting process is still in the range of $50 \mu \mathrm{m}$. Stealth dicing has shown great potential, but runs into problems if metallic layers on the wafer surface occur. In a water-jet guided laser cutting process, it could be rather difficult not to damage the polymer tape, which typically extends below the wafer.

According to the literature the regime of ultra-short pulses starts at pulse duration of less than a few picoseconds. But it is not completely known which pulse duration below this threshold delivers the best results in terms of quality and productivity. Thus in this paper the influence of the pulse duration in a range of $200 \mathrm{fs}$ up to $10 \mathrm{ps}$ on the cutting process of thin silicon is investigated.

\section{Experimental setup and approach}

The experiments are carried out with a Light Conversion Pharos with various pulse durations between 200 fs and $10 \mathrm{ps}$. The laser is operated at the second harmonic wavelength of $514 \mathrm{~nm}$ and a repetition rate of $200 \mathrm{kHz}$. A raw beam diameter of $5 \mathrm{~mm}$ and a focussing optics with $100 \mathrm{~mm}$ focussing length leads to a focal spot diameter of $15 \mu \mathrm{m}$. The beam deflection is realized by a Scanlab IntelliScan 14DE galvo scanner. The polarization of the beam is linear with a contrast ratio of 1:300. The direction of the polarisation is set perpendicular to the cutting / scanning direction by means of a half-wave-plate.

Figure 1 shows the spot diameter in the focal plane for three different pulse durations: $200 \mathrm{fs}, 1 \mathrm{ps}$ and $10 \mathrm{ps}$. The images are recorded with a $1,67 \mu \mathrm{m}$ pixel size CMOS camera from $\mu$ eye. For all pulse durations the spot diameter 
amounts $15 \mu \mathrm{m}\left(1 / \mathrm{e}^{2}\right)$ and the beam profile is without any deviations. So influences from different pulse durations on the beam profile can be excluded.
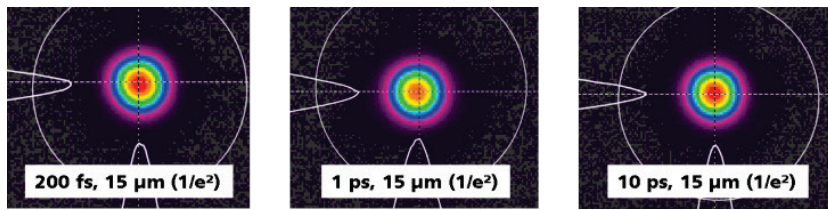

Figure 1 Spot diameter, measured with 1,67 $\mu \mathrm{m}$ pixel CMOS camera at three different pulse durations

In order to investigate the cutting geometry within the experiments, $500 \mu \mathrm{m}$ thick boron doped silicon wafers are firstly grooved with certain experimental parameters and afterwards cleaved perpendicularly. By this the cutting width and depth can be measured quite easily and furthermore possible material damage next to the cutting kerf could be observed. After the cleaving the specimens are polished to increase the measurement accuracy.

The left side of figure 2 shows this approach. The silicon is monocrystalline with an orientation of 100.
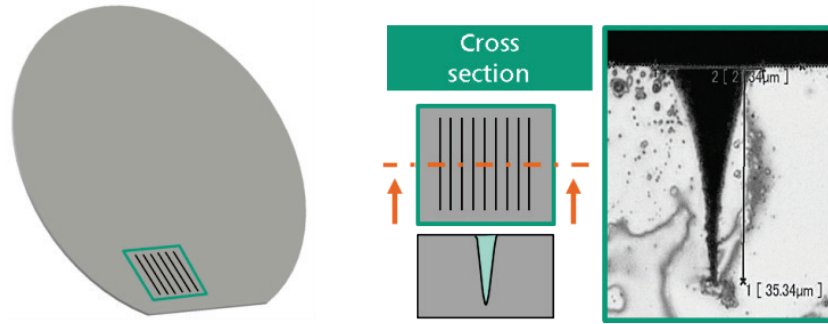

Figure 2 Experimental approach and setup. $500 \mu \mathrm{m}$ wafer are grooved with different parameters and afterwards cleaved perpendicularly. Used laser source is a Light Conversion Pharos and scanner a Scanlab IntelliScan 14de

During the experiments the following parameters are varied and the influence on the cutting geometry is analyzed:

- Pulse duration: $0,2 / 1 / 10 \mathrm{ps}$

- Pulse energy: 7,5/10/12,5 $\mu \mathrm{J}$

- Peak fluence: $8,5 / 11,3 / 14,2 \mathrm{~J} / \mathrm{cm}^{2}$

- Scanning speed: $0,5 / 1 / 1,5 / 2 \mathrm{~m} / \mathrm{s}$

- Pulse spacing: 2,5 / 5 / 7,5 / $10 \mu \mathrm{m}$

- Passes: $100 / 500 / 750 / 1000$

\section{Experimental results}

Firstly the ablation threshold for pulse durations of $10 \mathrm{ps}, 1$ ps and $200 \mathrm{fs}$ is determined with the method of Liu [1]. During the method the diameter of the ablated geometry is measured, squared and plotted against the peak fluence in logarithmic scale. Figure 3 shows a Liu-diagram and on the right side exemplary pictures of the ablation at the different pulse durations at $1 \mathrm{~J} / \mathrm{cm}^{2}$. Although it turns out that the ablation threshold fluence is almost independent from the pulse duration between $10 \mathrm{ps}$ and $200 \mathrm{fs}$, the ablation geometry looks different. At 10 ps the surface is quite rough and shows possibly a solidified melting layer. With decreased pulse durations of $1 \mathrm{ps} / 200$ fs the surface seems to be smoother and the ablation is characterized by a sharp ring.

We explain the difference behavior with the relation between pulse duration and thermal interaction time of elec- trons and lattice. According to the two-temperature-model, the photon energy is absorbed by the electrons and afterwards, depending on certain material parameters, transferred to the actual material. This interaction time is in the range of $1 \mathrm{ps}$ for silicon [8]. So we expect lower thermal impact for $1 \mathrm{ps}$ and $200 \mathrm{fs}$ laser pulses, since the energy transfer starts after the pulse is already gone.
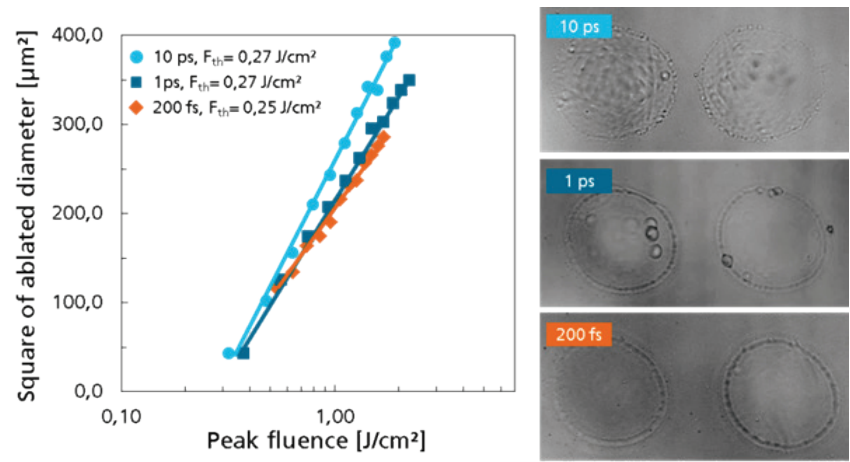

Figure 3 Diagram with linear regression of square of ablated diameter and peak fluence according to Liu [1]; Exemplary pictures of ablated geometry at $10 \mathrm{ps}, 1 \mathrm{ps}$ and $200 \mathrm{fs}$ at a fluence of $1 \mathrm{~J} / \mathrm{cm}^{2}$, recorded with a laser scanning microscope

Different pulse energies are investigated as to the cutting depth attainable. At a pulse repetition frequency of 200 $\mathrm{kHz}$, the scanning speed is set to values of $0,5 \mathrm{~m} / \mathrm{s}$ and 2 $\mathrm{m} / \mathrm{s}$. Figure 4 shows ablation results for different pulse energies - 7.5, 10 and $12.5 \mu \mathrm{J}$ - for up to 1000 repeats. In general it is shown that the cutting depth is strongly depending on the pulse energy

At a pulse energy of 7,5 $\mu \mathrm{J}$ and $0,5 \mathrm{~m} / \mathrm{s}$ scanning speed, the cutting depth amounts to $80 \mu \mathrm{m}$ after 500 repetitions and does not rise significantly during the following 500 repetitions. For an increased pulse energy level of $12,5 \mu \mathrm{J}$, the cutting depth after 1000 repetitions is about $125 \mu \mathrm{m}$ but again the development of the cutting depth shows a saturation behavior. The groove width also strongly depends on the pulse energy. For a low pulse energy, the width amounts less than $30 \mu \mathrm{m}$. At medium and high fluences the groove dimensions increase to $35-40 \mu \mathrm{m}$. The correlation between cutting width and pulse energy can be explained with the increasing effective diameter with growing pulse energy.

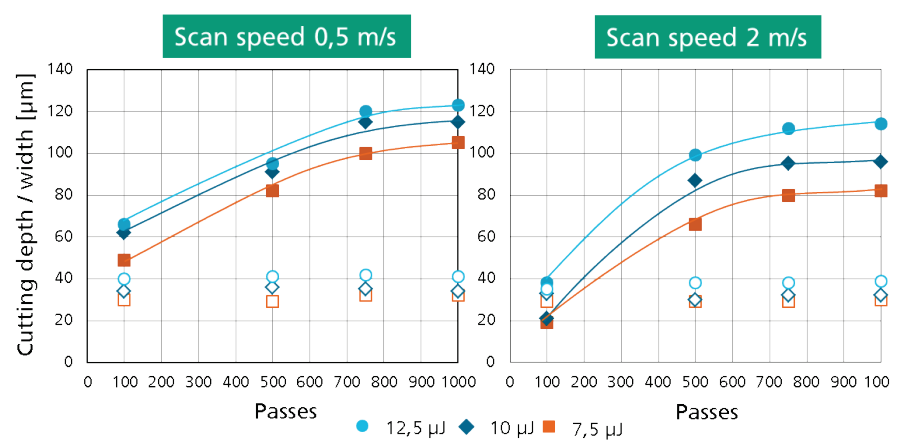

Figure 4 Cutting depth and width depending on pulse energy and scanning speed at a pulse duration of $10 \mathrm{ps}$. Solid symbols indicate the cutting depth and open symbols the cutting width

A similar behavior can be obtained also for a higher scanning speed of $2 \mathrm{~m} / \mathrm{s}$. Due to the larger pulse distance, less pulses are distributed on the same area and therefore the thermal impact in the cutting kerf is smaller. The final cut- 
ting depth for $12,5 \mu \mathrm{J}$ amounts $117 \mu \mathrm{m}$ and for $7,5 \mu \mathrm{J} 80$ $\mu \mathrm{m}$. The cutting width is also a little bit smaller, but not significant.

Figure 5 shows exactly the same kind of experiments as before, with the exception that the pulse duration is decreased by a factor of 50 to $200 \mathrm{fs}$, which means at the same level of pulse energy a 50 times higher pulse peak power. For both scanning speeds the development of the cutting depth is similar to the 10 ps experiments. This means that the ablation mechanism is the same. After having a closer look, it turns out that the final cutting depth is always deeper for $200 \mathrm{fs}$ and also the effective cutting speed (scanning speed / \# passes) is higher. At $2 \mathrm{~m} / \mathrm{s}$ and $12,5 \mu \mathrm{J}$ pulse energy the cutting depth amounts $130 \mu \mathrm{m}$. Compared to 10 ps this means an increasement of $15 \mu \mathrm{m}$ or $13 \%$.

Moreover the cutting depth is significantly smaller and not so strongly depending on the pulse energy. Obviously the optical power is transmitted effectively deep into the ablation groove, instead of being absorbed on the work piece surface. Even for 12,5 $\mu \mathrm{J}$ pulse energy the cutting kerf is not larger than $25 \mu \mathrm{m}$ at $2 \mathrm{~m} / \mathrm{s}$. For $10 \mathrm{ps}$ and this kind of parameter combination a width of almost $40 \mu \mathrm{m}$ is measured.

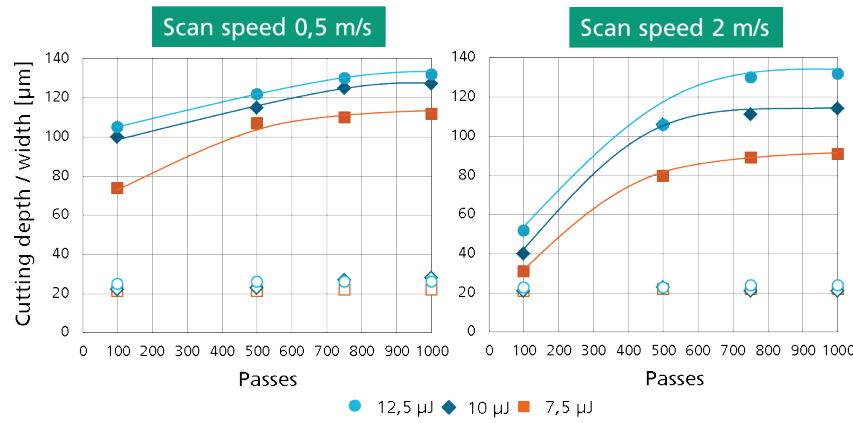

Figure 5 Cutting depth and width depending on pulse energy and scanning speed at a pulse duration of $200 \mathrm{fs}$. Solid symbols indicate the cutting depth and open symbols the cutting width

Figure 6 shows a cutting edge of full cut silicon chip with a thickness of $120 \mu \mathrm{m}$. The material is the same as before, but the thickness is reduced to obtain a complete cut instead of only grooves. The effective cutting speed amounts $12 \mathrm{~mm} / \mathrm{s}$, resulting from a scanning speed of $25 \mathrm{~m} / \mathrm{s}$ and ca. 2000 passes. The high scanning speed of $25 \mathrm{~m} / \mathrm{s}$ was realized by a polygon scanner head. One can see a thin film of sticking ablation particles in the cutting kerf and also at the edge. This shows that the surrounding material is only affected very near to the surface.

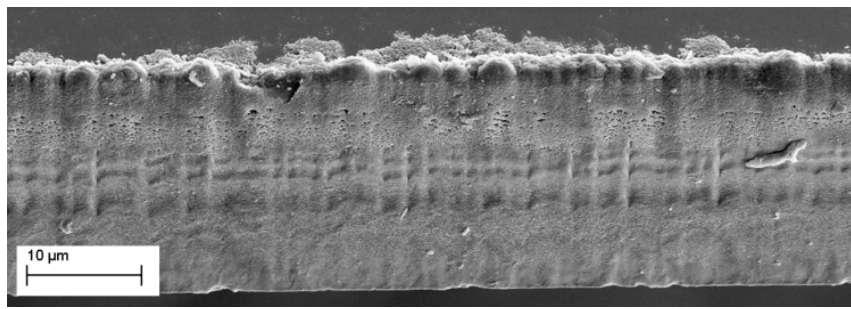

Figure 6 Cutting edge from a laser-cut $120 \mu \mathrm{m}$ thick silicon wafer after 2000 consecutive passes

During the experiments we also made the experience that a certain amount of the debris can be washed away in an additional cleaning step in an ultrasonic bath filled with etha- nol. The wavelike lines parallel to the cutting edge indicate the cutting progress over the passes. With increasing depth in the cutting kerf the surface quality gets smoother and less ablation particles are to be found.

In addition figure 7 shows the exit cutting edge. Therefore the specimen was flipped $180^{\circ}$. The picture reveals a very homogenous surface structure with a measured roughness of around $1 \mu \mathrm{m}$. The cutting edge is very straight and sharp. No cracks could be identified, which are usually very critical in terms of the maximal breaking strength of the chip. Even a very thin and sensitive gold layer on the backside is not damaged during the ablation cutting process. This shows how gentle and precisely the singulation process takes place.

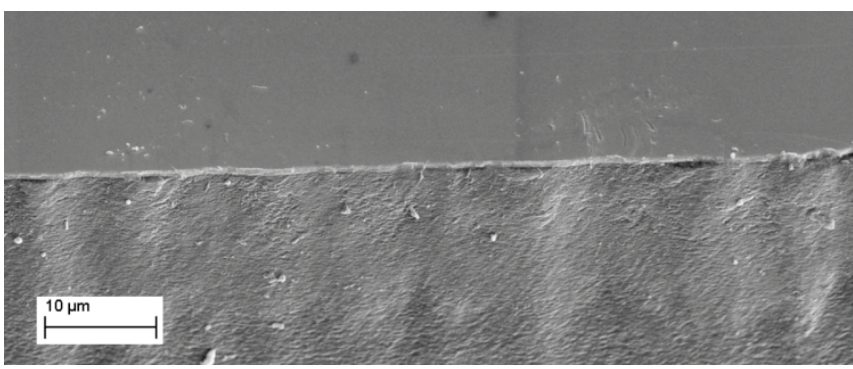

Figure 7 Exit cutting edge from a laser-cut $120 \mu \mathrm{m}$ thick silicon wafer after 2000 consecutive passes

\section{Discussion}

The cutting kerf geometry strongly depends on the fluence level or pulse energy. A higher fluence leads to deeper grooves, but also to an increased width. The progress of the depth is characterized by a saturation level, which is determined by the particular fluence. This behavior is affected by a multi-pass ablation strategy using a Gaussian beam profile together with the material properties of silicon or semiconductors in general. In a multi-pass ablation strategy, the beam is guided over the workpiece several times. In every repetition a certain amount of material is removed out of the cutting groove, Figure 8. During the first repetition the Gaussian beam hits a flat surface perpendicularly. Thus, the complete fluence or intensity of the beam is available for the ablation process. The geometry corresponds to the intensity profile of the beam, and the biggest amount of material is ablated at the center position because of the highest fluence or intensity.

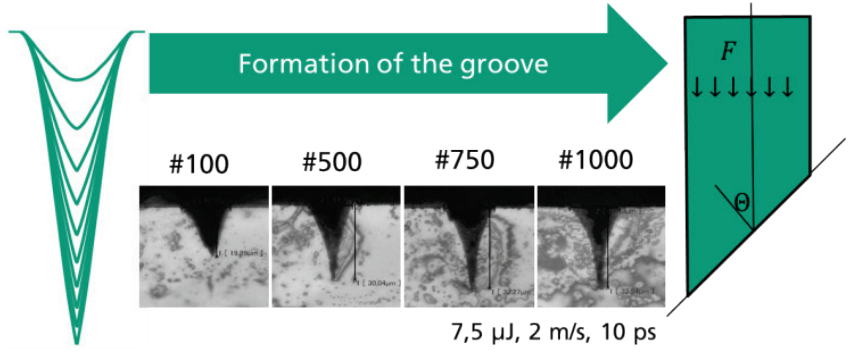

Figure 8 Development of the cutting kerf depending on the projected fluency

After the first repetition, the irradiated area is no longer planar, and the incoming laser beam is projected to the walls of the cutting kerf. Therefore, the effective beam diameter increases, and the fluence or intensity decreases. Figure 9 shows the calculation of an ablation geometry and 
the dependency of the projected intensity from the angle of incidence. These calculations and diagrams are taken from [9].

The differential equation for the ablation depth reads:

$$
\begin{gathered}
\frac{d z}{d r}=\sqrt{\left(\frac{F(r)}{F_{t h}}\right)^{2}-1} \cdot \theta\left(\frac{F(r)}{F_{t h}}-1\right) \\
\text { with } \theta(x)=1 \text { if } x \geq 0 \\
\theta(x)=0 \text { if } x<0
\end{gathered}
$$

The critical angle can be determined with the following equation:

$$
\varphi_{c r}=\arccos \left(\frac{F_{t h}}{F(r)}\right)
$$

With the previously determined value of the ablation threshold, it is now possible to calculate a critical angle for certain pulse energies. $A_{\text {Spot,critical }}$ is in this context the area which is at least necessary to overcome the ablation threshold in terms of the fluence. $A_{\text {Spot }}$ is the actual beam cross-sectional area, determined by the beam diameter of $15 \mu \mathrm{m}$.

$$
\begin{array}{r}
F_{\text {th }}=0.27 \frac{\mathrm{J}}{\mathrm{cm}^{2}}=\frac{2 \cdot E_{P}}{A_{\text {Spot,critical }}} \\
\text { with } E_{P}=7.5 \mu \mathrm{J} \\
\text { follows } A_{\text {Spot,critical }}=5.5 \cdot 10^{-5} \mathrm{~cm}^{2} \\
\text { with } A_{\text {Spot }}=2.424 \cdot 10^{-5} \mathrm{~cm}^{2} \\
\text { follows } \varphi_{\text {critical }}=\arccos \frac{A_{\text {Spot }}}{A_{\text {Spot, critical }}}=63,85^{\circ} .
\end{array}
$$

The equation shows that the critical angle is directly related to the pulse energy. For example, the critical angle for 10 $\mu \mathrm{J}$ increases to $70,1^{\circ}$ and for $12.5 \mu \mathrm{J}$ to $74,6^{\circ}$. These critical angles determine the attainable cutting depth in relation to the cutting width.
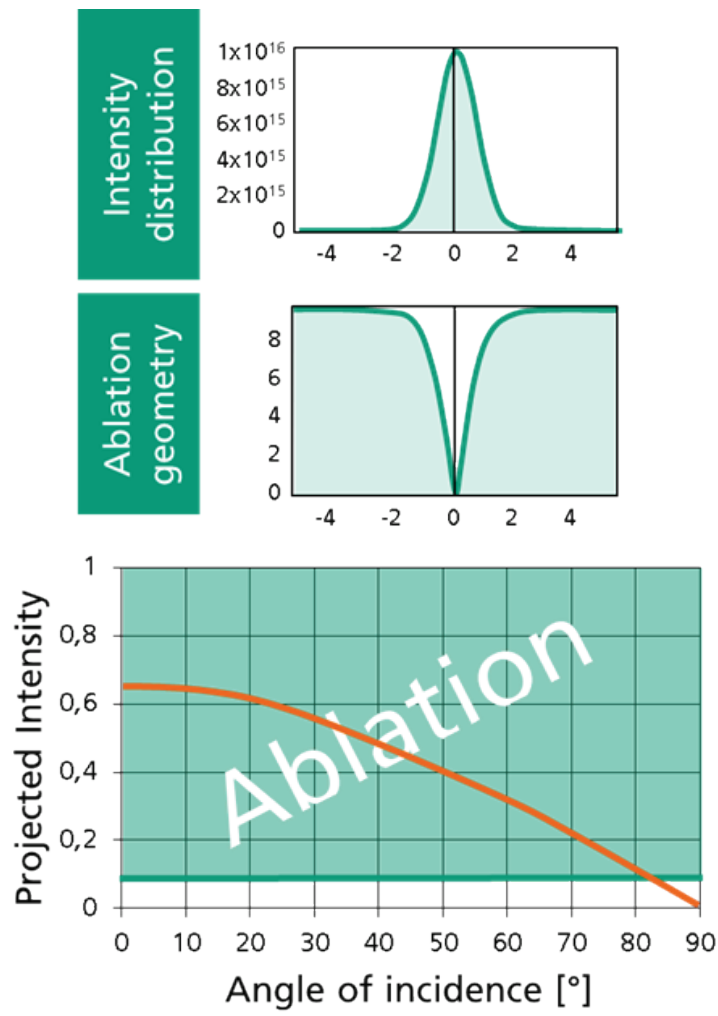

Figure 9: Explanation of the cutting kerf geometry and the progress of the groove depth due to the Gaussian beam profile
Obviously the laser pulse duration also plays an important role, since a significant difference in the cutting geometry can be measured at different pulse durations. Shorter pulse durations lead to deeper and narrower grooves. So the ablation seems to be more selective. From our point of view the $50 \mathrm{x}$ times shorter pulse duration leads to a shift of the energy transfer between photons and electrons to a time slot completely after the actual pulse. In contrast to a pulse duration of a few picoseconds, no interaction between laser radiation and vapour or melt takes place. The material is also heated up faster and thus less material melts, but is directly vaporized.

\section{Summary}

Dicing of silicon wafers with an ultra-short pulsed laser system has been investigated as to which cutting depths and kerf widths can be reached. The experiments were carried out using a frequency doubled Light Conversion Pharos Laser with a wavelength of $514 \mathrm{~nm}$ and variable pulse duration between 200 fs and 15 ps. A spot size of $15 \mu \mathrm{m}$ is attained by using a $100 \mathrm{~mm} \mathrm{f}$-Theta lens.

According to the experiments, the amount of the groove depth strongly depends on the laser fluence. The depth of the kerf develops logarithmically with a saturation level after a certain depth or number of passes. Afterwards the depth no longer increases. Different pulse overlaps only influence the progress of the cutting depth, but not the final amount.

This correlation is the result of a multi-pass ablation strategy with ultra-short pulsed laser radiation, in which a critical angle of incidence limits the attainable cutting geometry and depth. The critical angle is determined by ablation threshold of the material together with the incoming laser fluence. The largest amount of material is always removed in the center of the cutting kerf because of the peak intensity. Around the center a smaller intensity leads first to angled wall, second to insufficient laser fluence and finally to an ablation stop if the ablation threshold is not reached anymore.

The experiments also show a significant influence of the pulse duration on the cutting geometry, although the ablation thresholds are the same for $200 \mathrm{fs}, 1$ ps and 10 ps. A threshold of $0,27 \mathrm{~J} / \mathrm{cm}^{2}$ was measured with single spot ablation experiments on the surface of the silicon wafer. During the cutting experiments it turns out that the shortest pulse duration leads to much deeper and narrower grooves compared to a pulse duration of $10 \mathrm{ps}$. This behavior is independent from pulse energy and pulse overlap. We explain it with the 50x higher pulse peak intensity and therefore less heat conduction in the surrounding material and a very selective ablation process.

\section{Acknowledgments}

This work is funded from BMBF and supervised from VDI in the project SEMILAS

\section{References}

[1] Liu J M. Simple technique for measurements of pulsed Gaussian-beam spot sizes. Optics Letters, Vol. 7, Nr. 5, 1982 
[2] Schoenfelder S., Ebert M., Landesberger C., Bock K., Bagdahn J. 2007 Investigations of the influence of dicing techniques on the strength properties of thin silicon, Microelectronics Reliability 47 168-178 (2007)

[3] Crawford T.H.R., Borowiec A., Haugen H.K. 2005 Femtosecond laser micromachining of grooves in silicon with $800 \mathrm{~nm}$ pulses, Appl. Phys. A 80, 1717-1724 (2005)

[4] Bovatsek J M, Patel R S. Highest-speed dicing of thin silicon wafers with nanosecond-pulse $355 \mathrm{~nm}$ q-switched laser source using line-focus fluence optimization technique. Proc. SPIE 7585. Santa Clara, CA. 2010

[5] Masayoshi K, Naoki U, Etusji O, Ryuji S, Kazuhiro A, and Kenshi F. Advanced Dicing Technology for Semiconductor Wafer - Stealth Dicing. Semiconductor Manufacturing, VOL. 20, NO. 3, Taiwan. 2007

[6] Richerzhagen B, Industrial applications of the water-jet guided laser. The Industrial Laser User, Issue 28, Switzerland. 2003

[7] Thorstensen J., Foss E. Temperature dependent ablation threshold in silicon using ultrashort laser pulses, Appl. Phys. 112, 103514 (2012)

[8] Jeschke H., Garcia M., Lenzner M., Bonse J., Krüger J.,Kautek W. Laser ablation thresholds of silicon for different pulse durations: theory and experiment, Applied surface Science 197-198, 839-844, (2002)

[9] Eppelt. U., Simulation of ablation crater geometry of dielectrics and semiconductors after radiation after radiation with ultra-short pulsed lasers, PhD-thesis, RWTH Aachen, (2014)

(Received: July 10, 2014, Accepted: February 18, 2015) 